\title{
3 A family of distortion risk measures
}

Value at Risk (VaR) has been adopted as a standard tool to assess the risk and to calculate capital requirements in the insurance industry. As it has been shown in Chapter 1, VaR at level $\alpha$ is the $\alpha$-quantile of a random variable $X$ (which is often called loss). Recalling Definition 1.8

$$
\operatorname{VaR}_{\alpha}(X)=\inf \left\{x \mid F_{X}(x) \geqslant \alpha\right\}=F_{X}^{-1}(\alpha),
$$

where $F_{X}$ is the cumulative distribution function (cdf) of $X$ and $\alpha$ is the confidence or the tolerance level $0 \leqslant \alpha \leqslant 1$. However, VaR is known to present a number of pitfalls when applied in practice. A disadvantage when using $\mathrm{VaR}$ in the insurance or financial contexts is that the capital requirements for catastrophic losses based on this measure can be underestimated, i.e. the necessary reserves in adverse scenarios may well be less than they should be. The underestimation of capital requirements may be aggravated when fattailed losses are incorrectly modeled by mild-tailed distributions, such as the Normal distribution. There are attempts to overcome this kind of model risk when using VaR or, at least, to quantify the risk related to the modelling [Alexander and Sarabia, 2012]. But, in addition, a second drawback is that the VaR may fail the subadditivity property. As it has been shown in Table 1.2 of Chapter 1, a risk measure is subadditive when the aggregated risk is less than or equal to the sum of individual risks. Subadditvity is an appealing property when aggregating risks in order to preserve the benefits of diversification. VaR is subadditive for elliptically distributed losses [McNeil et al., 2005]. However, the subadditivity of VaR is not granted, as indicated in Artzner et al. [1999] and Acerbi and Tasche [2002].

Remember now Definition 1.9 from Chapter 1. In there Tail Value at Risk (TVaR) has been defined as

$$
\operatorname{TVaR}_{\alpha}(X)=\frac{1}{1-\alpha} \int_{\alpha}^{1} \operatorname{VaR}_{\lambda}(X) d \lambda
$$


Table 3.1 VaR95\% and TVaR95\% illustration

\begin{tabular}{rrr} 
& VaR95\% & TVaR95\% \\
\hline Empirical & 47.6 & 125.5 \\
Normal & 87.0 & 105.9 \\
Lognormal & 48.9 & 119.1 \\
\hline
\end{tabular}

* Cost of claims in thousands of Euro

Roughly speaking, the TVaR is understood as the mathematical expectation beyond VaR. The TVaR risk measure does not suffer the two drawbacks discussed above for VaR and, as such, would appear to be a more powerful measure for assessing the actual risks faced by insurance companies and financial institutions. However, TVaR has not been widely accepted by practitioners in the financial and insurance industry. VaR is currently the risk measure contemplated in the European solvency regulation for the insurance sector (Solvency II), and this is also the case of solvency regulation for the banking sector (Basel accords ${ }^{1}$ ). The TVaR measures average losses in the most adverse cases rather than just the minimum adverse loss, as the VaR does. Therefore, capital reserves based on the TVaR have to be considerably higher than those based on VaR and significant differences in the size of capital reserves can be obtained depending on which risk measure is adopted.

An illustration of the risk value obtained for the VaR $95 \%$ and TVaR $95 \%$ considering three alternative distributions is provided in Table 3.1. Note that huge differences in risk amounts can be obtained.

This chapter is motivated by the following question. Can a risk measure be devised that would provide a risk assessment that lies somewhere between those offered by the VaR and the TVaR? To this end, a new family of risk measures (GlueVaR) is proposed, which forms part of a wider class referred to as distortion risk measures.

GlueVaR risk measures are defined by means of a four-parameter function. By calibrating the parameters, GlueVaR risk measures can be matched to a wide variety of contexts. Specifically, once a confidence level has been fixed, the new family contains risk measures that lie between those of VaR and

1 Although it seems that changing VaR by TVaR with a lower confidence level is something that is really under consideration for regulatory capital requirements in the Banking sector. 
TVaR and which may adequately reflect the risk of mild-tailed distributed losses without having to resort to VaR. In certain situations, however, even more conservative risk measures than TVaR may be preferred. It is shown that these highly conservative risk measures can also be defined by means of the GlueVaR family. In order to preserve the benefits of diversification when aggregating risks, subadditivity is an appealing property of a risk measure. As it has been shown in Chapter 1 , the subadditivity property ensures that the risk measure value of the aggregated risk is lower than or equal to the sum of individual risk measure values. In this chapter the subadditivity property of GlueVaR risk measures is investigated.

\subsection{Overview on risk measures}

Two main groups of axiom-based risk measures are coherent risk measures, as stated by Artzner et al. [1999], and distortion risk measures, as introduced by Wang [1996]. Concavity of the distortion function is the key element to define risk measures that belong to both groups [Wang and Dhaene, 1998]. Suggestions on new desirable properties for distortion risk measures are proposed in Balbás et al. [2009], while generalizations of this kind of risk measures can be found, among others, in Hürlimann [2006] and $\mathrm{Wu}$ and Zhou [2006]. As shown in Goovaerts et al. [2012], it is possible to link distortion risk measures with other interesting families of risk measures developed in the literature.

The axiomatic setting for risk measures has extensively been developed since seminal papers on coherent risk measures and distortion risk measures. Each set of axioms for risk measures corresponds to a particular behavior of decision makers under risk, as it has been shown, for instance, in Bleichrodt and Eeckhoudt [2006] and Denuit et al. [2006]. Most often, articles on axiombased risk measurement present the link to a theoretical foundation of human behavior explicitly. For example, Wang [1996] shows the connection between distortion risk measures and Yaari's dual theory of choice under risk; Goovaerts et al. [2010] investigate the additivity of risk measures in Quiggin's rank-dependent utility theory; and Kaluszka and Krzeszowiec [2012] introduce the generalized Choquet integral premium principle and relate it to Kahneman and Tversky's cumulative prospect theory.

Many articles have appeared in recent years that pay attention to risk measures based on distortion functions or on generalizations of the quantiles. An example of the first group is Zhu and Li [2012]. Bellini and Gianin [2012] and Bellini et al. [2014] fit to second group. An interplay between both groups 
is found in Dhaene et al. [2012a] and Goovaerts et al. [2012].

\subsection{Distortion risk measures}

Distortion risk measures were introduced by Wang [Wang, 1995, 1996] and they are closely related to the distortion expectation theory [Yaari, 1987]. A review on how risk measures can be interpreted from several perspectives is provided in Tsanakas and Desli [2005], and a clarifying explanation of the relationship between distortion risk measures and distortion expectation theory is included. A detailed literature review of distortion risk measures is available in [Denuit et al., 2005; Balbás et al., 2009]. There are two key elements to define a distortion risk measure: first, the associated distortion function; and, second, the concept of the Choquet [Choquet, 1954] Integral. The distortion function, Choquet Integral and the distortion risk measure concepts can be defined as follows:

- Distortion function. Let $g:[0,1] \rightarrow[0,1]$ be a function such that $g(0)=$ $0, g(1)=1$ and $g$ is injective and non-decreasing. Then $g$ is called a distortion function.

- Choquet Integral. The (asymmetric) Choquet Integral with respect to a set function $\mu$ of a $\mu$-measurable function $X: \Omega \rightarrow \overline{\mathbb{R}}$ is denoted as $\int X d \mu$ and is equal to

$$
\int X d \mu=\int_{-\infty}^{0}\left[S_{\mu, X}(x)-\mu(\Omega)\right] d x+\int_{0}^{+\infty} S_{\mu, X}(x) d x,
$$

if $\mu(\Omega)<\infty$, where $S_{\mu, X}(x)=\mu(\{X>x\})$ denotes the survival function of $X$ with respect to $\mu$. Note that $\Omega$ denotes a set, which in financial and insurance applications is the sample space of a probability space. A set function $\mu$ in this context is a function defined from $2^{\Omega}$ (the set of all subsets of $\Omega$ ) to $\overline{\mathbb{R}}$. A $\mu$-measurable function $X$ is, widely speaking, a function defined on $\Omega$ such that expressions like $\mu(\{X>x\})$ or $\mu(\{X \leqslant x\})$ make sense. See Denneberg [1994] for more details.

- Distortion risk measure. Let $g$ be a distortion function. Consider a random variable $X$ and its survival function $S_{X}(x)=P(X>x)$. Then, function $\rho_{g}$ defined by $\rho_{g}(X)=\int_{-\infty}^{0}\left[g\left(S_{X}(x)\right)-1\right] d x+\int_{0}^{+\infty} g\left(S_{X}(x)\right) d x$ is called a distortion risk measure.

From the previous definitions, it is straightforward to see that for any random variable $X, \rho_{g}(X)$ is the Choquet Integral of $X$ with respect to the set 
function $\mu=g \circ P$, where $P$ is the probability function associated with the probability space in which $X$ is defined.

The mathematical expectation is a distortion risk measure whose distortion function is the identity function [Denuit et al., 2005], this is, $\rho_{\mathrm{id}}(X)=\mathbb{E}(X)$. Therefore, a straightforward way to interpret a distortion risk measure is as follows: first, the survival function of the random variable is distorted $\left(g \circ S_{X}\right)$; second, the mathematical expectation of the random variable with respect to this distorted probability is computed. From a theoretical point of view, note that this interpretation fits the discussion that risk may be defined as an expected value in many situations [Aven, 2012].

VaR and TVaR measures are in fact distortion risk measures. The associated distortion functions of these risk measures are shown in Table 3.2.

Table 3.2 VaR and TVaR distortion functions

\begin{tabular}{ll}
\hline Risk measure & Distortion function \\
\hline VaR & $\psi_{\alpha}(u)= \begin{cases}0 & \text { if } 0 \leqslant u<1-\alpha \\
1 & \text { if } 1-\alpha \leqslant u \leqslant 1,\end{cases}$ \\
TVaR & $\gamma_{\alpha}(u)= \begin{cases}\frac{u}{1-\alpha} & \text { if } 0 \leqslant u<1-\alpha \\
1 & \text { if } 1-\alpha \leqslant u \leqslant 1\end{cases}$ \\
\hline
\end{tabular}

For a confidence level $\alpha \in(0,1)$.

Based on the distortion functions shown in Table 3.2, once $\alpha$ is fixed it can be proved that $\operatorname{VaR}_{\alpha}(X) \leqslant \operatorname{TVaR}_{\alpha}(X)$ for any random variable $X$.

Remark 3.1. Let $g$ and $g^{*}$ be two distortion functions and let $\rho_{g}$ and $\rho_{g^{*}}$ be their respective distortion risk measures. Suppose that $g(u) \leqslant g^{*}(u)$ for all $u \in[0,1]$. Then $\rho_{g}(X) \leqslant \rho_{g^{*}}(X)$ for any random variable $X$.

This result follows immediately from the definition of distortion risk measures, because

$$
\begin{aligned}
\rho_{g}(X) & =\int_{-\infty}^{0}\left[g\left(S_{X}(x)\right)-1\right] d x+\int_{0}^{+\infty} g\left(S_{X}(x)\right) d x \\
& \leqslant \int_{-\infty}^{0}\left[g^{*}\left(S_{X}(x)\right)-1\right] d x+\int_{0}^{+\infty} g^{*}\left(S_{X}(x)\right) d x \\
& =\rho_{g^{*}}(X) .
\end{aligned}
$$


Many articles have recently examined risk measures based on either distortion functions [Zhu and Li, 2012; Belles-Sampera et al., 2013a, 2014a, 2016b; Guillen et al., 2016; Tsanakas and Millossovich, 2016] or generalizations of the quantiles [Bellini and Gianin, 2012; Bellini et al., 2014]. The interplay between both of these two groups of risk measures has been examined [Dhaene et al., 2012a; Goovaerts et al., 2012].

\subsection{A new family of risk measures: GlueVaR}

A new family of distortion risk measures, named GlueVaR, is here defined. Originally, we introduced this family in Belles-Sampera et al. [2014a]. The main reason for defining these GlueVaR risk measures is a response to the concerns expressed by risk managers regarding the choice of risk measures in the case of regulatory capital requirements. However, as it has been already mentioned, an axiomatic approach to define or represent risk measures is more frequent in the literature [Artzner et al., 1999; Föllmer and Schied, 2002; Frittelli and Rosazza Gianin, 2002; Denuit et al., 2006; Song and Yan, 2009; Cerreia-Vioglio et al., 2011; Ekeland et al., 2012; Goovaerts et al., 2012; Grechuk et al., 2012].

Any GlueVaR risk measure can be described by means of its distortion function. Given a confidence level $\alpha$, the distortion function for GlueVaR is:

$$
\kappa_{\beta, \alpha}^{h_{1}, h_{2}}(u)=\left\{\begin{array}{cl}
\frac{h_{1}}{1-\beta} \cdot u & \text { if } 0 \leqslant u<1-\beta \\
h_{1}+\frac{h_{2}-h_{1}}{\beta-\alpha} \cdot[u-(1-\beta)] & \text { if } 1-\beta \leqslant u<1-\alpha \\
1 & \text { if } 1-\alpha \leqslant u \leqslant 1
\end{array}\right.
$$

where $\alpha, \beta \in[0,1]$ such that $\alpha \leqslant \beta, h_{1} \in[0,1]$ and $h_{2} \in\left[h_{1}, 1\right]$. Parameter $\beta$ is the additional confidence level besides $\alpha$. The shape of the GlueVaR distortion function is determined by the distorted survival probabilities $h_{1}$ and $h_{2}$ at levels $1-\beta$ and $1-\alpha$, respectively. We call parameters $h_{1}$ and $h_{2}$ the heights of the distortion function.

A wide range of risk measures may be defined under this framework. Note that $\operatorname{VaR}_{\alpha}$ and $\operatorname{TVaR}_{\alpha}$ are particular cases of this new family of risk measures. Namely, $\operatorname{VaR}_{\alpha}$ and $\operatorname{TVaR}_{\alpha}$ correspond to distortion functions $\kappa_{\alpha, \alpha}^{0,0}$ and $\kappa_{\alpha, \alpha}^{1,1}$, respectively. By establishing suitable conditions on the heights $h_{1}$ and $h_{2}$, the GlueVaR family is very flexible. For example, risk managers might like to select $\alpha, \beta, h_{1}$ and $h_{2}$ so that

$$
\operatorname{VaR}_{\alpha}(X) \leqslant \operatorname{GlueVaR}_{\beta, \alpha}^{h_{1}, h_{2}}(X) \leqslant \operatorname{TVaR}_{\alpha}(X) .
$$


This can be achieved by selecting a set of parameters for their associated distortion functions to ensure that $\psi_{\alpha}(u) \leqslant \kappa_{\beta, \alpha}^{h_{1}, h_{2}}(u) \leqslant \gamma_{\alpha}(u)$ for any $u \in$ $[0,1]$, following remark 3.1, i.e. by forcing condition $h_{1} \leqslant \frac{1-\beta}{1-\alpha}$. An example of such a case is shown in Figure 3.1 (left-hand side).

The GlueVaR family also allows us to define a highly conservative risk measure GlueVaR ${ }_{\beta, \alpha}^{h_{1}, h_{2}}$, such that

$$
\operatorname{TVaR}_{\alpha}(X) \leqslant \operatorname{GlueVaR}_{\beta, \alpha}^{h_{1}, h_{2}}(X) \leqslant \operatorname{TVaR}_{\beta}(X)
$$

for any $X$ and that the associated distortion function $\kappa_{\beta, \alpha}^{h_{1}, h_{2}}$ is concave in $[0,1]$. In this case, $\frac{1-\beta}{1-\alpha} \leqslant h_{1}$ and $h_{2}=1$ must be fulfilled, as occurs in the example shown in Figure 3.1 (right-hand side).

Figure 3.1 Examples of GlueVaR distortion functions.

Left. Distortion function is concave in $[0,1-\alpha)$ and $\operatorname{VaR}_{\alpha}(X) \leqslant \operatorname{GlueVaR}_{\beta, \alpha}^{h_{1}, h_{2}}(X) \leqslant$ $\operatorname{TVaR}_{\alpha}(X)$ for a random variable $X$;

Right. Distortion function is concave in the whole range $[0,1]$ and $\operatorname{TVaR}_{\alpha}(X) \leqslant$ $\operatorname{GlueVaR}_{\beta, \alpha}^{h_{1}, h_{2}}(X) \leqslant \operatorname{TVaR}_{\beta}(X)$ for a random variable $X$.
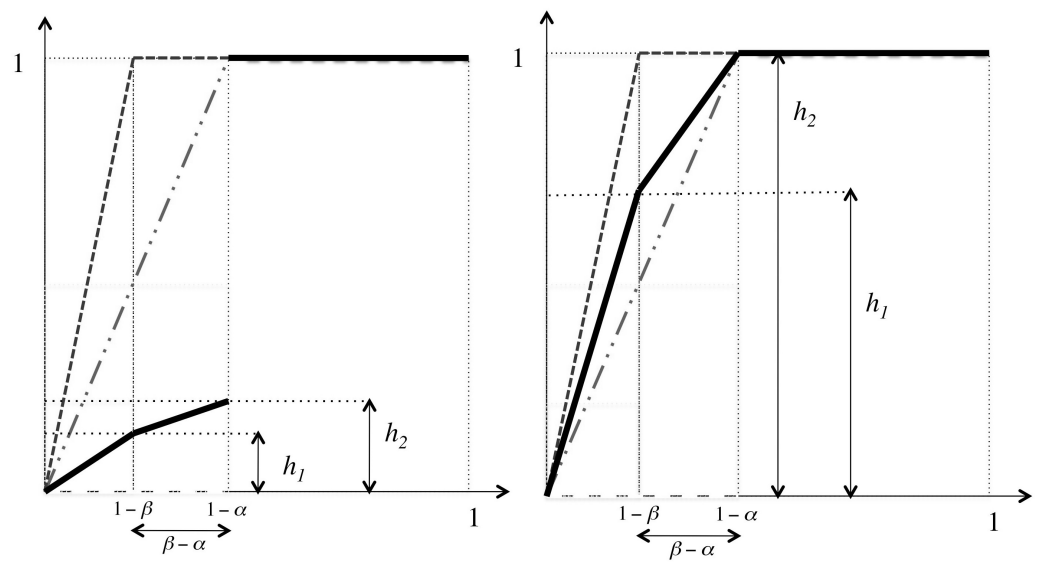

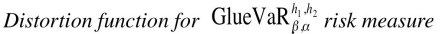

- . Distortion function for $\mathrm{TVaR}_{\alpha}$ risk measure Distortion function for $\mathrm{TVaR}_{\beta}$ risk measure - - Distortion function for $\mathrm{VaR}_{\alpha}$ risk measure

\subsection{Linear combination of risk measures}

Given a random variable $X$ and for fixed tolerance levels $\alpha$ and $\beta$ so that $\alpha<$ $\beta$, GlueVaR $h_{\beta, \alpha}^{h_{1}, h_{2}}(X)$ can be expressed as a linear combination of $\operatorname{TVaR}_{\beta}(X)$, 
$\operatorname{TVaR}_{\alpha}(X)$ and $\operatorname{VaR}_{\alpha}(X)$. This result allows us to translate the graphicalbased construction of GlueVaR risk measures into an algebraic construction based on standard risk measures.

If the following notation is used,

$$
\left\{\begin{array}{l}
\omega_{1}=h_{1}-\frac{\left(h_{2}-h_{1}\right) \cdot(1-\beta)}{\beta-\alpha} \\
\omega_{2}=\frac{h_{2}-h_{1}}{\beta-\alpha} \cdot(1-\alpha) \\
\omega_{3}=1-\omega_{1}-\omega_{2}=1-h_{2}
\end{array}\right.
$$

then the distortion function $\kappa_{\beta, \alpha}^{h_{1}, h_{2}}(u)$ in (3.1) may be rewritten as (details can be found in Section A.1 of the Appendix):

$$
\kappa_{\beta, \alpha}^{h_{1}, h_{2}}(u)=\omega_{1} \cdot \gamma_{\beta}(u)+\omega_{2} \cdot \gamma_{\alpha}(u)+\omega_{3} \cdot \psi_{\alpha}(u)
$$

where $\gamma_{\beta}, \gamma_{\alpha}, \psi_{\alpha}$ are the distortion functions of TVaR at confidence levels $\beta$ and $\alpha$ and of VaR at confidence level $\alpha$, respectively (see Table 3.2). Therefore GlueVaR is a risk measure that can be expressed as a linear combination of three risk measures: TVaR at confidence levels $\beta$ and $\alpha$ and VaR at confidence level $\alpha$,

$$
\operatorname{GlueVaR}_{\beta, \alpha}^{h_{1}, h_{2}}(X)=\omega_{1} \cdot \operatorname{TVaR}_{\beta}(X)+\omega_{2} \cdot \operatorname{TVaR}_{\alpha}(X)+\omega_{3} \cdot \operatorname{VaR}_{\alpha}(X)
$$

Given this relationship, an alternative notation for $\operatorname{GlueVaR}_{\beta, \alpha}^{h_{1}, h_{2}}(X)$ and its related distortion function can be used. The notation $\operatorname{GlueVaR}_{\beta, \alpha}^{\omega_{1}, \omega_{2}}(X)$ or $\kappa_{\beta, \alpha}^{\omega_{1}, \omega_{2}}(u)$ may, on occasions, be preferred to that based on heights $h_{1}$ and $h_{2}$. The bijective relationship between pairs $\left(h_{1}, h_{2}\right)$ and $\left(\omega_{1}, \omega_{2}\right)$ is also shown in Section A.2 of the Appendix.

Specifically, in order to simplify the statement of Proposition 4.1, the expression of $\kappa_{\beta, \alpha}^{\omega_{1}, \omega_{2}}(u)$ is

$$
\kappa_{\beta, \alpha}^{\omega_{1}, \omega_{2}}(u)= \begin{cases}{\left[\frac{\omega_{1}}{1-\beta}+\frac{\omega_{2}}{1-\alpha}\right] \cdot u} & \text { if } 0 \leqslant u<1-\beta \\ \omega_{1}+\frac{\omega_{2}}{1-\alpha} \cdot u & \text { if } 1-\beta \leqslant u<1-\alpha \\ 1 & \text { if } 1-\alpha \leqslant u \leqslant 1\end{cases}
$$

An interesting interpretation of (3.4) in the context of decision making and risk management is that GlueVaR risk measures arise as a linear combination of three possible scenarios. So, two levels of severity tolerance can be 
fixed, namely $\alpha$ and $\beta$, with $\alpha<\beta$. Then, the risk can be measured in the highly conservative scenario with TVaR at level $\beta$; in the conservative scenario with TVaR at level $\alpha$; and in the less conservative scenario with VaR at level $\alpha$.

Each combination of these risk scenarios reflects a concrete risk attitude. Therefore, it can be said that the combination of these risk scenarios in this context is something that is directly identified by an explicit GlueVaR risk measure. To some extent, these risk attitudes could be related to risk appetite [Aven, 2013].

From the practitioner's point of view, four parameters must be fixed in order to define the GlueVaR risk measure. The $\alpha$ and $\beta$ values correspond to the confidence levels used for bad and very bad scenarios, respectively. For instance, $\alpha=95 \%$ and $\beta=99.5 \%$ could be selected, which are equivalent to one bad event every twenty years or one bad event every two hundred, respectively. The other two parameters are directly related to the weights given to these scenarios. For instance, it could be said that the three components of GlueVaR in expression (3.4) are equally important. This would imply $\omega_{1}=\omega_{2}=\omega_{3}=1 / 3$, so the corresponding $h_{1}$ and $h_{2}$ parameters could be found. When $\omega_{1}=\omega_{2}=\omega_{3}=1 / 3$ and $\alpha=95 \%, \beta=99.5 \%$, these parameters are $h_{1}=11 / 30$ and $h_{2}=2 / 3$.

\subsection{Subadditivity}

In a seminal article [Artzner et al., 1999] the following set of axioms that a risk measure should satisfy was established: positive homogeneity, translation invariance, monotonicity and subadditivity. Authors referred to such risk measures as coherent riskmeasures. Distortion risk measures always satisfy the first three properties, but subadditivity is only guaranteed when the distortion function is concave [Denneberg, 1994; Wang and Dhaene, 1998; Wirch and Hardy, 2002]. Therefore, VaR, unlike TVaR, is not coherent. In some situations, coherence of risk measures is a requirement [Cox, 2012] but, nonetheless, some criticisms can be found [Dhaene et al., 2008]. Additional properties for distortion risk measures are provided in [Jiang, 2008; Balbás et al., 2009], which may complement the list of properties for risk measures shown in Tables 1.2 and 1.4 of Chapter 1. In this section we focus on the subadditivity property.

In order to preserve the benefits of diversification when aggregating risks, subadditivity is an appealing property of a risk measure. As it has been shown in Chapter 1, the subadditivity property ensures that the risk mea- 
sure value of the aggregated risk is lower than or equal to the sum of individual risk measure values. For distortion risk measures, subadditivity may be defined as follows.

Definition 3.1. Given a confidence level $\alpha \in[0,1]$, a distortion risk measure $\rho_{g}$ is subadditive if, for any pair $X, Y$,

$$
\int(X+Y) d(g \circ P) \leqslant \int X d(g \circ P)+\int Y d(g \circ P),
$$

where the integral symbol stands for Choquet Integrals with respect to the set function $g \circ P$.

The Choquet integral condition used in the definition can be rewritten, in terms of survival functions and Lebesgue integrals, as

$$
\int_{0}^{+\infty} g\left(S_{X+Y}(z)\right) d z \leqslant \int_{0}^{+\infty} g\left(S_{X}(x)\right) d x+\int_{0}^{+\infty} g\left(S_{Y}(y)\right) d y .
$$

As shown, GlueVaR risk measures may be interpreted as a linear combination of VaR and TVaR risk measures. Therefore, a GlueVaR risk measure is coherent when the weight assigned to $\mathrm{VaR}$ is zero and the weights of the $\mathrm{TVaR}_{\alpha}$ and $\mathrm{TVaR}_{\beta}$ are non-negative. In terms of the parameters of the distortion function, GlueVaR is subadditive (and thus coherent) if $h_{2}=1$ and $\frac{1-\beta}{1-\alpha} \leqslant h_{1}$. More generally, any property satisfied by TVaR but not by VaR will be inherited by GlueVaR if $\omega_{1} \geqslant 0$ and $\omega_{3}=0$ in expression (3.2).

Subaddtitivity in the whole domain is a strong condition. When dealing with fat tail losses (i.e. low-frequency and large-loss events), risk managers are especially interested in the tail region. Fat right-tails have been extensively studied in insurance and finance [Wang, 1998; Embrechts et al., 20o9a,b; Degen et al., 2010; Nam et al., 2011; Chen et al., 2012] and the behavior of aggregate risks in the tail region has received huge attention by researchers in last years [Cheung, 2009; Song and Yan, 2009; Hua and Joe, 2012]. To the best of our knowledge, however, previous studies of the subadditivity of risk measures in the tail region are scarce [Danielsson et al., 2005; Hua and Joe, 2012].

\subsection{Concavity of the distortion function}

The subadditivity characteristic in the whole domain is in general not satisfied by GlueVaR risk measures. It was showed that GlueVaR risk measures 
can be interpreted as a linear combination of a highly conservative scenario, a conservative scenario and a less conservative scenario. We argued that a particular risk attitude is reflected depending on how these scenarios are weighted.

Given $\alpha$ and $\beta$, the other two parameters are directly related to the weights given to these scenarios. The shaded areas in Figure 3.2 delimit feasible weights $\left(\omega_{1}, \omega_{2}\right)$ for GlueVaR $\mathrm{R}_{\beta, \alpha}^{\omega_{1}, \omega_{2}}$. For instance, it could be said that the three components of GlueVaR in expression (3.4) are equally important, that is, $\omega_{1}=\omega_{2}=\omega_{3}=1 / 3$. The point $(1 / 3,1 / 3)$ in Figure 3.2 corresponds to the balanced risk attitude on the part of risk managers when faced by the three components shown in (3.4). The corresponding distortion function $\kappa_{\beta, \alpha}^{\omega_{1}, \omega_{2}}$ is concave on $[0,1-\alpha)$ in the lightly shaded area. Yet, the distortion function is not concave on $[0,1-\alpha)$ in the darkly shaded area. The distortion function is concave in $[0,1]$ in the boldest continuous segment and, thus, the associated GlueVaR risk measure is subadditive.

Note that any pair of weights $\left(\omega_{1}, \omega_{2}\right)$ on the boldest line in Figure 3.2 leads to $\omega_{3}=0$. This means that a zero weight is allocated to the least conservative scenario, i.e. the one associated with the $\operatorname{VaR}_{\alpha}(X)$. This is indicative of the decision makers' conservative approach. Nonetheless, differences in just how restrictive this conservative attitude is can be found among the weights lying on this line: the nearer to $\left(\omega_{1}, \omega_{2}\right)=\left(\frac{\beta-1}{\beta-\alpha}, \frac{1-\alpha}{\beta-\alpha}\right)$, the less restrictive it is, while the nearer to $\left(\omega_{1}, \omega_{2}\right)=(1,0)$, the more conservative it is.

If $\omega_{1}<0$, risk managers are optimistic regarding the impossibility of the occurrence of the worst case scenario, and so attach a negative weight to it.

\subsection{Example of risk measurement with GlueVaR}

Data for the cost of claims involving three type of damages described in the previous chapter are used to illustrate the application of GlueVaR risk measures in risk measurement. The sample consists of $n=350$ observations of the cost of individual claims in thousands of euros. In Table $3.3 \mathrm{a}$ set of quantile-based risk measures including three different GlueVaR are displayed. The table displays the corresponding risk figures for the cost of claims for property damage $\left(X_{1}\right)$, the cost of claims of bodily injuries $\left(X_{2}\right)$, the cost of claims of medical expenses $\left(X_{3}\right)$ and the aggregate cost of claims $\left(X_{1}+X_{2}+X_{3}\right)$.

The selection of the three GlueVaR risk measures included in Table 3.3 de- 
Figure 3.2 Given $\alpha$ and $\beta$, the shaded areas delimits feasible weights $\left(\omega_{1}, \omega_{2}\right)$ for GlueVaR $\boldsymbol{\beta}_{\boldsymbol{\beta}, \boldsymbol{\alpha}}^{\omega_{1}, \omega_{2}}$.

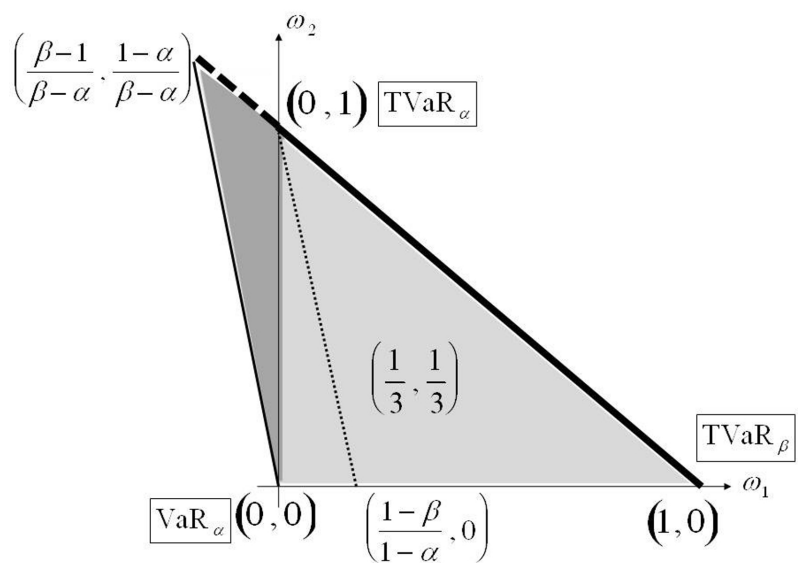

serves further explanation. The two confidence levels considered are $\alpha=$ $95 \%$ and $\beta=99.5 \%$. The heights $\left(h_{1}, h_{2}\right)$ are $(11 / 30,2 / 3),(0,1)$ and $(1 / 20$, $1 / 8$ ) respectively. Different attitudes in front of the three scenarios of risk assessment are represented. GlueVaR ${ }_{99.5 \%, 95 \%}^{11 / 30,2 / 3}$ corresponds to a balanced attitude because the three quantile-based risk measures TVaR99.5\%, TVaR $95 \%$ and $\mathrm{VaR}_{95 \%}$ are equally important, i.e. $\omega_{1}=\omega_{2}=\omega_{3}=1 / 3$. A different attitude is symbolized by GlueVaR $\mathrm{R}_{99.5 \%, 95 \%}^{0,1}$ with associated weights $\omega_{1}=-1 / 9$, $\omega_{2}=10 / 9$ and $\omega_{3}=0$. It corresponds to a scenario in which the manager overweights $\mathrm{TVaR}_{95 \%}$ and allocates the lowest feasible weight to TVaR99.5\% given that a zero weight is allocated to $\mathrm{VaR}_{95 \%}$. Finally, GlueVaR $\mathrm{R}_{99.5 \%, 95 \%}^{1 / 20,1 / 8}$ reflects a risk measurement attitude just a bit more conservative than the one represented by using $\mathrm{VaR}_{95 \%}$, assigning low weights to $\mathrm{TVaR}_{99.5 \%}$ and $\operatorname{TVaR}_{95 \%}\left(\omega_{1}=1 / 24\right.$ and $\left.\omega_{2}=1 / 12\right)$.

As it is shown in Table 3.3, GlueVaR $\mathrm{R}_{99.5 \%, 95 \%}^{11 / 30,2 / 3}$ is more conservative than the other two selected GlueVaR risk measures. This result can be generalized to all situations because the associated distortion function of GlueVaR $\mathrm{R}_{99.5 \%, 95 \%}^{11 / 30,2 / 3}$ is greater than the other two distortion functions in the whole domain. Note that it is also observed in Table 3.3 that

$$
\text { GlueVaR }_{99.5 \%, 95 \%}^{1 / 20,1 / 8} \leqslant \text { GlueVaR }_{99.5 \%, 95 \%}^{0,1} \cdot
$$

It is only valid to these data and an ordering between them can not be generalized. However, a relationship between these two GlueVaR risk measures 
Table 3.3 Quantile-based risk measures and subadditivity

\begin{tabular}{lrrrrr}
\hline & $\mathbf{X}_{\mathbf{1}}$ & $\mathbf{X}_{\mathbf{2}}$ & $\mathbf{X}_{\mathbf{3}}$ & $\mathbf{X}_{\mathbf{1}}+\mathbf{X}_{\mathbf{2}}+\mathbf{X}_{\mathbf{3}}$ Difference $^{(*)}$ \\
& (a) & (b) & (c) & (d) & $(\mathbf{a}+\mathrm{b}+\mathrm{c}-\mathrm{d})$ \\
\hline VaR95\% $_{95}$ & 2.5 & 0.6 & 1.1 & 5.9 & -1.7 \\
TVaR $_{95 \%}$ & 12.5 & 8.0 & 1.3 & 19.7 & 2.1 \\
TVaR $_{99.5 \%}$ & 40.8 & 42.1 & 1.8 & 81.1 & 3.6 \\
GlueVaR $_{99.5 \%, 95 \%}^{11 / 30,2 / 3}$ & 18.6 & 16.9 & 1.4 & 35.6 & 1.9 \\
GlueVaR $_{99.5 \%, 95 \%}^{1 / 10,8}$ & 4.9 & 2.9 & 1.1 & 10.2 & -1.3 \\
GlueVaR $_{99.5 \%, 95 \%}^{0,1}$ & 9.4 & 4.2 & 1.2 & 12.9 & 1.9 \\
\hline
\end{tabular}

(*) Benefit of diversification.

and quantile-based risk measures can be established. It has been shown that

$$
\operatorname{VaR}_{\alpha} \leqslant \operatorname{GlueVaR}_{\beta, \alpha}^{h_{1}, h_{2}} \leqslant \mathrm{TVaR}_{\alpha} \text { if } h_{1} \leqslant \frac{1-\beta}{1-\alpha} .
$$

That means,

$$
\begin{aligned}
& \operatorname{VaR}_{95 \%} \leqslant \operatorname{GlueVaR}_{99.5 \%, 95 \%}^{0,1} \leqslant \mathrm{TVaR}_{95 \%}, \text { because } 0 \leqslant 0.1 \text {, and } \\
& \operatorname{VaR}_{95 \%} \leqslant \operatorname{GlueVaR}_{99.5 \%, 95 \%}^{1 / 20,1 / 8} \leqslant \mathrm{TVaR}_{95 \%} \text {, because } 0.05 \leqslant 0.1 .
\end{aligned}
$$

Although results in Table 3.3 may suggest that

$$
\mathrm{TVaR}_{95 \%} \leqslant \operatorname{GlueVaR}_{99.5 \%, 95 \%}^{11 / 30,2 / 3} \leqslant \mathrm{TVaR}_{99.5 \%},
$$

this can not be asserted in general because conditions on the parameters of the GlueVaR risk measure to satisfy

$$
\begin{gathered}
\mathrm{TVaR}_{\alpha} \leqslant \mathrm{GlueVaR}_{\beta, \alpha}^{h_{1}, h_{2}} \leqslant \mathrm{TVaR}_{\beta} \\
\text { are } \frac{1-\beta}{1-\alpha} \leqslant h_{1} \text { and } h_{2}=1 .
\end{gathered}
$$

In this case it holds that $0.1 \leqslant 0.37$ but $h_{2} \neq 1$. 
Let us analyze the subadditivity property. Like the VaR95\%, note that the GlueVaR ${ }_{99.5 \%, 95 \%}^{1 / 20,1 / 8}$ fails to be subadditive for $X_{1}, X_{2}$ and $X_{3}$ since $4.9+2.9+$ $1.1<10.2$. Let us emphasize that these three GlueVaR risk measures have not associated a concave distortion function in the whole domain, so the subadditivity property can not be guaranteed for any of them. The fact that risk values are subadditive for the GlueVaR ${ }_{99.5 \%, 95 \%}^{11 / 30,2 / 3}$ and GlueVaR $\mathrm{P}_{99.5 \%, 95 \%}^{0,1}$ is a characteristic attributable to this data but cannot be generalized to all contexts. In the case of the GlueVaR $\mathrm{R}_{99.5 \%, 95 \%}^{11 / 30,2 / 3}$ and $\mathrm{GlueVaR}_{99.5 \%, 95 \%}^{1 / 20,1 / 8}$, the associated distortion function is concave in $[0,1-\alpha)$.

\subsection{Exercises}

1. Consider the following empirical distribution

$$
13,15,26,26,26,37,37,100
$$

Determine the GlueVaR $\mathrm{R}_{85 \%, 50 \%}^{11 / 30,2 / 3}$ and GlueVaR ${ }_{85 \%, 50 \%}^{0,1}$.

Hint: The heights $\left(h_{1}, h_{2}\right)$ equal to $(11 / 30,2 / 3)$ and $(0,1)$ correspond to $\left(\omega_{1}=1 / 3, \omega_{2}=1 / 3\right)$ and $\left(\omega_{1}=-1 / 9, \omega_{2}=10 / 9\right)$, respectively.

2. Consider the following distribution function $F(x)=\frac{x^{2}}{9}$ for $0<x \leqslant 3$. Find the GlueVaR $\mathrm{R}_{99.5 \%, 95 \%}^{1 / 20,1 / 8}$.

Hint: The heights $\left(h_{1}, h_{2}\right)$ equal to $(1 / 20,1 / 8)$ correspond to $\omega_{1}=1 / 24$ and $\omega_{2}=1 / 12$.

3. (Exemple 6.7 in [McNeil et al., 2005]) Consider a defaultable corporate bond. The default probability is equal to $2 \%$. The current price of the bond is 100. If there is no default, a bond pays in $t+1$ an amount of 105 , otherwise there is no payment. Hence $L$ the loss of bond is equal to 100 when the bond defaults and to -5 otherwise. Compute the $V_{a R} R_{95}$ of the following two portfolios:

- Portfolio A consists of 100 units of this bond.

- Portfolio B consists of 100 independent bonds with the same characteristics that this bond.

Hint: The loss function of a bond can be expressed as $L=100 Y-5(1-$ $Y)=105 Y-5$, where $Y$ is a indicator variable that takes value 1 if the bond defaults and 0 otherwise. 
4. Let $X$ and $Y$ be two independent random variables uniformly distributed between $[0,1]$ and $Z=X+Y$ their sum. Analyze if it holds that $\operatorname{VaR}_{25 \%}$ $(Z)<\operatorname{VaR}_{25 \%}\left(X_{1}\right)+\operatorname{VaR}_{25 \%}\left(X_{2}\right)$.

Hint: The cumulative distribution function of $Z$ is

$$
F_{Z}(z)=\left\{\begin{array}{cc}
\frac{z^{2}}{2} \quad \text { if } 0<z \leqslant 1 \\
-\frac{z^{2}}{2}+2 z-1 \text { if } 1<z \leqslant 2
\end{array}\right.
$$


